\title{
CÓMO DESARROLLAR LA CIENCIA Y LA TECNOLOGÍA EN LOS PAÍSES ATRASADOS: Una mirada en prospectiva
}

\author{
Federico Dejo Soto \\ PROFESOR CONTRATADO DE ESAN \\ MÁSTER EN PlanificACión Y MEdio AMBIENTE
}

\section{Resumen}

El presente trabajo expone, a criterio del autor, cuáles son las condiciones necesarias que deben confluir para hacer posible un impulso permanente al desarrollo científico y tecnológico en los países atrasados. Se trabaja la idea central de que el desarrollo de la ciencia y tecnología depende de las posibilidades de cambio y progreso de otros sectores de la vida económica, social y cultural de un país. Entre estas condiciones se analizan la educación, el desarrollo industrial, el vínculo con las necesidades de la economía y el bienestar de la población, la retribución digna al científico, el surgimiento de una masa crítica de científicos, el partir de lo ya avanzado, la mentalidad libre y audaz, el manejo de idiomas extranjeros, la masificación del uso de las tecnologías de información y la política de apoyo a las publicaciones.

\section{Introducción}

Cuando se debate sobre el tema del desarrollo de la ciencia y tecnología, se constata fácilmente que existe una marcada asociación entre éste y el grado de industrialización de un país. Mientras más industrializado es un país, mayor es también su potencial en el campo del desarrollo científico y tecnológico; y a la inversa, mientras menos industrializado es un país, también es menor su desarrollo en esos campos del saber humano.
Ante esta evidencia, la explicación más aceptada, pero a la vez más simple, sostiene que en el caso de los países más adelantados ello se debería al apoyo permanente del Estado, que invierte una proporción significativa de sus recursos económicos en el campo de la educación, la investigación y otros servicios de alta tecnología. Y en el caso de los países menos adelantados, además de la escasez de recursos, ocurriría que hay poca comprensión de la importancia de desarrollar esos campos, pues se tiende a aceptar la exis- 
tencia de un especie de asignación de roles en la división mundial del trabajo, en virtud de la cual a los países ricos les tocaría cumplir el papel de líderes e invertir grandes montos en ciencia y tecnología. Por lo tanto, en el caso de los países pobres, dada las limitaciones que soportan, se justificaría la reducida inversión. Y como la brecha entre países ricos y países pobres crece de año en año, es cada vez más lo que puede invertir un país industrializado en ciencia y tecnología en comparación con lo que pueden invertir los países pobres. Esta situación haría que, con el transcurso del tiempo, la diferencia entre lo que puede hacer en investigación y desarrollo un país rico frente a uno pobre sea mayor. Entonces, en las condiciones actuales, la posibilidad de que los países subdesarrollados lleguen a igualar a los países ahora más desarrollados es prácticamente nula, constatación que en algunos casos conduce a aceptar este hecho como si fuera una ventaja comparativa de nuestros países, que no tendrían que hacer los gastos que en esos rubros hacen los países ricos. Es decir, supuestamente todos nos beneficiaríamos del gasto que hacen los países industrializados, a pesar de que no invertimos en ello. Al aceptar este supuesto, los países pobres se están atando las manos para conseguir el progreso de sus pueblos.

En realidad, invertir en investigación y tecnología es uno de los requisitos básicos para que un país subdesarrollado logre su desarrollo económico autosostenido y competitivo frente al resto del mundo, se haga menos dependiente de la tecnología de los países más avanzados y, por lo tanto, sea capaz de crear valor agregado y mucha más riqueza a fin de mejorar las condiciones de vida de su población. Por ello, en las líneas siguientes se expone un conjunto de condiciones indispensables que un país tipificado como pobre debe reunir para que pueda desarrollarse en el campo de la ciencia y tecnología. Pero hay que poner énfasis en que estas condiciones implican ciertos cambios en la estructura económica, social, cultural, educativa e incluso política de los países que quieren salir del subdesarrollo. En efecto, estos hechos han ocurrido con anterioridad al acelerado crecimiento de los países industrializados y han facilitado el camino a la investigación y desarro1lo. Es decir, la hipótesis que aquí se expone es que de nada valdría que un país pobre invierta, de repente, gran parte de su presupuesto público en proyectos de investigación si paralelamente no se han producido otros cambios estructurales que forman el contexto dentro de los cuales pueden tener éxito las actividades científico y tecnológicas.

En síntesis, el punto de vista que se expone en las siguientes líneas, si bien no deja de considerar como aspecto muy importante el apoyo consciente del Estado a la Investigación y Desarrollo Tecnológico (I+D), busca argumentar en el sentido de que éste no es suficiente. Para que los esfuerzos de inversión rindan frutos, tienen que hacerse en el marco de un conjunto de otros elementos necesarios que los preceden y forman el contexto propicio.

\section{Condiciones necesarias que deben acompañar a un proceso de desarrollo en ciencia y tecnología}

\section{a) Lograr una educación escolar masiva y de calidad}

Mientras menor sea el analfabetismo y mayor la escolaridad en la población de 
un país, más factible será alcanzar logros en el campo de la investigación y el desarrollo. Es decir, una masa considerable de población cuyo nivel educativo le permita acceder a estudios universitarios y, como consecuencia de ello, dominar disciplinas científicas especializadas se convierte en un requisito para ir logrando un grupo sólido de profesionales con capacidad para desarrollar labores de investigación científica pura y aplicada. En consecuencia, uno de los requisitos básicos del impulso al desarrollo de la ciencia y tecnología es masificar la formación escolar y postescolar, apuntando a que los jóvenes desarrollen el interés por la investigación y la creatividad aplicada a los requerimientos de la vida diaria y, especialmente, de su entorno social y natural. Obviamente, a fin de lograr ese interés entre los jóvenes la educación debe ser también de calidad. Esto significa que debe conseguirse el objetivo de enseñar a razonar antes que a memorizar, así como desarrollar en los educandos las habilidades necesarias para que sean capaces de llevar a la práctica o a la experimentación sus propias ideas, antes que repetir mecánicamente lo aprendido en los textos.

Conviene recordar que el conocimiento científico es propio de la evolución del pensamiento humano y, por lo tanto, puede surgir en cualquier sociedad que haya logrado un grado mínimo de capacidad para sistematizar la realidad (sea natural o social) y elaborar pensamientos abstractos que le faciliten el desarrollo de razonamientos complejos, lo que conduce a la construcción de teorías científicas basadas en la experimentación y el contraste de hipótesis. Y la educación masiva en sus diversas modalidades es un poderoso vehículo que resulta en la mejora de las capacidades cognoscitivas de la población.
Es decir, las posibilidades del desarrollo científico son innatas y pueden darse en cualquier lugar del planeta sin distingo de razas. Más aún a inicios del siglo XXI, cuando la globalización del mundo a través de los medios de información computarizados permite la integración del conocimiento casi al instante y desde cualquier lugar del planeta. Los obstáculos de acceso a nuevos conocimientos por la dificultad debida a la distancia y el tiempo se están reduciendo. Y es la educación escolar y postescolar la que permite llevar los conocimientos científicos a amplios grupos sociales.

Pero conviene reiterar que las posibilidades de ese desarrollo serán mayores en la medida en que el acceso a la educación sea masivo y, en consecuencia, beneficie al mayor número de pobladores de una nación. Más aún, mientras más altos sean los niveles de estudios a los que puedan llegar los habitantes de un país, mejores serán las condiciones para que se produzca un desarrollo sostenido de la investigación en ciencia y tecnología. Los datos estadísticos que relacionan niveles de escolaridad con desarrollo científico y tecnológico demuestran que hay una fuerte asociación positiva entre estos aspectos. No por casualidad los países con mejores niveles de escolaridad y formación postescolar alcanzan también los mejores niveles de desarrollo en los campos del saber.

\section{b) La inversión en ciencia y tecnología aumenta cuando paralelamente hay desarrollo industrial}

La investigación científica, al basarse en procesos experimentales -de pruebaerror-prueba o de aproximaciones sucesivas a través del error y la prueba- que pue- 
den durar periodos muy largos, requiere por lo general un alto monto de inversión en recursos materiales y humanos que no puede ser solventado directamente por los investigadores. Por ello es necesaria la participación de instituciones públicas o privadas -empresas, fundaciones, universidades, etc.- con suficiente capacidad financiera para apoyar proyectos de investigación destinados al desarrollo de las ciencias básicas o aplicadas.

Pero dado que la capacidad financiera de un país está en función de la generación de riqueza o valor agregado por parte de su industria y servicios, la investigación es más factible en los países con mayor desarrollo industrial. Por lo tanto, mientras más industrializado sea un país, mayor tiende a ser la inversión que realiza en investigación. Las estadísticas señalan que los países con mayor desarrollo industrial invierten una proporción más alta de su PBI en ciencia y tecnología. Por esta razón, liberar las fuerzas de la economía industrial es un factor importante para estimular ese desarrollo.

El impulso a la ciencia y tecnología en un país no tiene futuro si no se dan medidas de política económica que posibiliten el crecimiento industrial y la ampliación del mercado interno. En otras palabras, la investigación encuentra estímulo cuando hay un fuerte dinamismo económico en el campo de la industria, el comercio y los servicios, pues son éstos los sectores de la economía que comienzan a demandar investigaciones específicas para mejorar sus productos o crear otros nuevos que satisfagan la demanda potencial o efectiva de sus clientes. Se crea así un círculo virtuoso en el cual el desarrollo científico y el desarrollo de los diferentes sectores de la economía se apo- yan y condicionan mutuamente, avanzando constantemente hacia mayores niveles.

\section{c) Retribuir con salarios dignos a quienes se dedican a la ciencia y la investigación}

Por su naturaleza, la labor de investigación requiere dedicación plena y generalmente exclusiva para obtener resultados en plazos relativamente cortos. Por ello, exige un sueldo decoroso que permita al investigador vivir sin sobresaltos debidos a carencias económicas y, además, mantenerse actualizado en los últimos avances científicos respecto a su tema de interés.

Mientras los países subdesarrollados no garanticen condiciones de trabajo dignas a sus científicos, se producirá de manera constante la emigración o fuga de talentos hacia los países desarrollados, sobre todo, donde ellos posiblemente encuentren oportunidades de realización profesional. Hay también quienes se quedan en sus países de origen, pero desempeñándose muchas veces en actividades totalmente ajenas a aquellas en las cuales se especializaron e invirtieron años de estudio y manutención. Es decir, se produce una pérdida paulatina del capital científico.

Cabe señalar que en los países desarrollados o en aquellos que se están esforzando constantemente para lograr el desarrollo, los investigadores científicos son considerados un capital estratégico y, por lo tanto, hay preocupación por darles un estatus socioeconómico por encima del correspondiente al promedio de los profesionales del país. En los últimos años, hemos podido apreciar cómo científicos de la ex Unión Soviética, ante la tremenda crisis económica que agobia a esa par- 
te del mundo, iniciaron un proceso de emigración hacia otros países en mejor situación, donde fueron rápidamente acogidos. Asimismo, puede mencionarse lo ocurrido durante la Segunda Guerra Mundial, cuando científicos alemanes opuestos al Nazismo fueron atraídos hacia Estados Unidos, que les ofrecía condiciones adecuadas para su labor. Y luego de la derrota alemana, los países victoriosos, sobre todo Estados Unidos y la entonces Unión Soviética, reclutaron a los más destacados científicos del país vencido para que trabajaran en los proyectos de Postguerra, principalmente en los relacionados con la energía atómica y la carrera espacial.

Entonces, si se busca retener a la comunidad científica no basta con apelar al recurso de la identidad nacional o la responsabilidad moral del investigador con su país, cuando en la práctica no se le proporciona los medios necesarios para que pueda desempeñarse con tranquilidad. Tal parece ser el caso del Perú, donde las pocas instituciones de investigación apoyadas por el Estado pagan a sus investigadores remuneraciones que no alcanzan ni siquiera para solventar los gastos de una canasta básica familiar. También sucede en las universidades públicas, donde la investigación y la publicación de obras de interés científico han venido disminuyendo por falta de presupuesto. En un trabajo realizado por quien esto escribe sobre cómo ha evolucionado la situación de la docencia en las universidades de Lima Metropolitana entre 1987 y 1993, se comparan los resultados de una misma encuesta aplicada a los docentes e investigadores universitarios en esos dos años. Los resultados demuestran cómo ha crecido el desinterés por las actividades científicas en paralelo con el empeoramiento de la situación económica del docente.

\section{d) Las actividades científicas de un país deben tener por objetivo contribuir a resolver necesidades de los diferentes rubros de la economía y del bienestar de la población}

A fin de que la investigación sea exitosa, debe haber una relación directa entre los temas que se investigan y la utilidad de los mismos en el campo del desarrollo tecnológico destinado a resolver problemas cruciales de la población o de los sectores productivos -los que a su vez inciden en la economía-. Es decir, a fin de que contribuya al desarrollo de un país, la investigación científica debe impactar en la generación de nuevas formas de ahorro, creación de riqueza o satisfacción de necesidades humanas urgentes. De no ser así, se estarían perdiendo recursos en proyectos que podrían ser banales para el interés de una nación. Por lo tanto, una adecuada relación entre ciencia y tecnología y desarrollo requiere de un diagnóstico previo de las principales demandas de investigación del país en los diferentes sectores del quehacer nacional, de modo que la atención de esas demandas causen impactos positivos en la producción de tecnologías que, a su vez, generen riqueza.

Generalmente, lograr una adecuada articulación entre esos factores en países como el nuestro, donde los recursos son escasos, hace necesaria la elaboración de un Plan Nacional de Investigación para el Desarrollo, en el que confluyan tanto el interés público como el privado en el apoyo a las actividades de investigación. Por ejemplo, es conocido que en los países altamente industrializados las universidades públicas reciben fuertes subvenciones de grandes empresas privadas para investigar sobre aspectos que, si bien son casi 
siempre de interés comercial, también son de interés colectivo y causan impacto económico y social. Pero también el Estado, a través de las instituciones normativas, puede opinar o tomar la iniciativa sobre la pertinencia o no pertinencia de dichos trabajos en función del interés nacional o la ética científica

Un aspecto poco explotado por los países subdesarrollados, y que les podría redituar importantes dividendos económicos, es el relacionado con la investigación biotecnológica. Un aprovechamiento más científico de sus potencialidades naturales, como el patentar el origen y uso de las variedades genéticas en animales y plantas oriundas, que luego son demandadas por el mercado internacional, podría significarles considerables ingresos en su balanza comercial. Mejor aun si, con un adecuado conocimiento y manejo de esos recursos biogenéticos, se desarrolla una industria alimentaria o de medicamentos que dé valor agregado a los recursos naturales. Se crearía entonces riqueza, parte de la cual se reinvertiría en ciencia y tecnología, con lo cual se cerraría el círculo virtuoso.

Pero para que ello ocurra, debe reiterarse la necesidad de un Plan de Investigación para el Desarrollo, que ordene aquellos rubros o sectores de la realidad que queremos conocer mejor a fin de convertirlos en una fuente de bienestar, trabajo y valor agregado.

\section{e) Debe crearse una comunidad científica amplia y de calidad}

No se puede realizar investigación científica de trascendencia si no se cuenta con un número suficiente de científicos cuya labor pueda potenciarse en el intercambio de ideas y los diversos métodos de expe- rimentación. En concreto, para desarrollar en forma significativa la investigación es necesario crear una comunidad científica que pueda trascender en el quehacer nacional.

Una manera de crear una comunidad científica es dando facilidades orientadas a que los graduados universitarios interesados en la investigación puedan acceder a estudios de postgrado a nivel de maestrías y doctorados. Por lo general, la obtención de un grado de doctor implica una mejor preparación para afrontar retos de investigación científica y tecnológica, así como para publicar artículos que difunden hipótesis o conocimientos nuevos.

Los indicadores sobre investigación y desarrollo indican que en los países industrializados la proporción de doctores por número de habitantes es mucho más alta que en el caso de los países subdesarrollados. Esto significa que las posibilidades de acceder a ese grado académico en las diferentes ramas del saber son mayores para la población de los países más adelantados. Este hecho facilita la conformación de un numeroso estrato de científicos con alta preparación académica interesados en la investigación, una comunidad científica de alto valor que encuentra en su actividad no sólo una forma de realización vocacional, sino también una forma de vida que los identifica.

Esa comunidad científica tiende, además, a organizarse por ramas de actividad o interés, propiciando el intercambio de ideas, hipótesis o exposición de resultados de investigaciones, lo que estimula y potencia la labor de investigación y contribuye al desarrollo de sus países. Por ejemplo, donde existe una fuerte comunidad de científicos son frecuentes los se- 
minarios, talleres, conferencias, mesas redondas, en las que se dan a conocer avances individuales o se analiza preocupaciones comunes buscándoles soluciones. Estos encuentros implican la existencia de una masa crítica que produce y genera flujos importantes de comunicación e información. En los países subdesarrollados, estas actividades son escasas precisamente por lo pequeño de la comunidad científica.

Queda claro entonces que en los países subdesarrollados se hace necesario contar con un número significativo de científicos que hayan obtenido altos grados académicos en las diversas especialidades de las ciencias básicas y aplicadas, pues de esa masa crítica puede provenir la mayor parte de las ideas creativas y las labores experimentales.

f) Se debe partir de los conocimientos más avanzados para desarrollarlos y/o mejorarlos

Es errónea la creencia de que, por su atraso, los países subdesarrollados no pueden acercarse a niveles de investigación parecidos a los de los países industrializados. Pero aunque parezca paradójico, a fin de que esa distancia se acorte es necesario partir de los niveles científicos que los países más industrializados ya han logrado, para tener posibilidades de competencia tanto en la producción de bienes como en conocimientos.

Si un país subdesarrollado pretende iniciarse en el arduo camino de la investigación y desarrollo tratando de descubrir por su cuenta todo lo que otros países ya conocen, posiblemente nunca llegará a alcanzarlos. La experiencia moderna enseña que los países subdesarrollados que han tenido éxito en lograr un nivel acelerado de desarrollo han podido hacerlo, en gran medida, porque precisamente han convocado a las empresas internacionales de mayor desarrollo tecnológico para que inviertan en sus economías o establezcan empresas subsidiarias que sirvan como modelo a fin de transferir conocimientos y tecnologías. Éstos han sido los casos de los llamados tigres asiáticos, como Hong Kong, Singapur, Corea del Sur, Taiwan y, últimamente, China continental.

La inversión de los grandes conglomerados internacionales en los países subdesarrollados supone la aplicación de tecnologías modernas que sirven de modelo y punto de partida para las industrias de capital nativo, las que paulatinamente comienzan a generar sus nuevos productos imitando, primero, y buscando superar, después, a las que les sirvieron de guía. Y en este tema los científicos nacionales tienen un importante rol que cumplir.

Ésta es una oportunidad importante y válida que los países atrasados deben saber aprovechar. Sin embargo, frente a esta posibilidad hay la opinión contraria de quienes señalan que los países subdesarrollados no deberían invertir en aquellos sectores de la economía donde los países industrializados ya son fuertes y les han sacado ventaja, como la industria automotriz o de computadoras. Esta postura recomienda, más bien, que se debe continuar con la explotación y exportación de los recursos primarios que hacen falta en los países desarrollados. Éste es un error que condena a nuestros países a no intentar incursionar en aquellos campos de la producción que concentra lo más avanzado de la tecnología y la ciencia aplicada. Si Corea del Sur hubiera desistido de desarrollar la industria automotriz o la de 
artefactos eléctricos porque la misma ya estaba desarrollada en Estados Unidos, Japón o Europa Occidental, posiblemente hoy en día se encontraría en una situación similar a la de la mayoría de los países tercermundistas. Suerte parecida hubiera corrido Taiwan si sus dirigentes e intelectuales hubieran pensado que no tenía sentido invertir en la industria de equipos e implementos de computadoras, o de maquinaria eléctrica liviana, entre otros rubros de su ahora poderosa economía. Consciente de lo importante de desarrollar productos de la industria de punta en tecnología, la China ha abierto sus puertas a las transnacionales para que inviertan y trasladen ahí sus tecnologías; y les permite, además, que obtengan sus ganancias al más puro estilo de las economías capitalistas.

\section{g) Debe desarrollarse una mentalidad científica libre, audaz y creativa}

Para que un país logre crear una comunidad científica creativa es necesario desarrollar una nueva actitud mental en el sistema educativo, en general, y en la comunidad científica, en particular.

Para ser creativo e innovador hay que atreverse constantemente a romper esquemas. Sólo las mentes abiertas pueden ver aquello que no alcanzan a vislumbrar las mentes que observan la realidad en forma estereotipada. En este sentido, la mente de un científico no puede estar sujeta a prejuicios que la hagan subjetiva y le impidan analizar con lucidez la realidad.

Por ello, crear mentalidad y actitud científica implica también dejar de lado intencionalidades políticas o religiosas al momento de investigar. Es decir, para avanzar y aprovechar la creatividad, la investigación debe ser libre, sin ataduras que la condicionen, salvo aquellas que imponen la ética y el respeto a la naturaleza y dignidad humanas.

Precisamente la actitud audaz ha permitido avances enormes en el campo de la cirugía: transplante de órganos, utilización de rayos láser, uso de cámaras de televisión diminutas dentro del organismo con las cuales se opera sin necesidad de cortes traumáticos.

Pero una investigación libre requiere también de regímenes políticos adecuados que le permitan desarrollarse. Un intelectual o científico es por excelencia un librepensador, pero si en la sociedad donde vive se le condiciona o limita, buscará la huída o simplemente se abstendrá de pensar. En regímenes totalitarios como los que se sufrieron en la Alemania de Hitler o en la ex Unión Soviética, eran frecuentes las disidencias de científicos que debieron emprender la fuga por no estar de acuerdo con los condicionamientos que les imponía el sistema político. Y poco a poco, eso fue minando el impulso al desarrollo en otros campos del saber y de la tecnología que favorecían el bienestar de las personas. Sin embargo, bajo ese tipo de regímenes sí se desarrollaron aquellos conocimientos relacionados con el armamentismo, el genocidio y la justificación del sistema político totalitario y opresivo.

\section{h) Los científicos y profesionales deben manejar los idiomas más usados en la comunidad científica internacional}

El desarrollo de la ciencia y la tecnología requiere de un flujo muy fuerte de información y comunicación entre científicos. Pero ocurre que por la predominancia de 
los países más industrializados, los conocimientos más avanzados están expuestos en sus lenguas de origen. Sin embargo, desde inicios de este siglo, el idioma inglés se ha venido consolidando como una especie de idioma universal, de modo que incluso se ha convertido en casi un segundo idioma en aquellos países industrializados europeos cuya lengua nativa es otra. El conocimiento del inglés les da una gran ventaja para ampliar rápidamente su comunicación e información con los conocimientos procedentes de Estados Unidos, la máxima potencia mundial.

No cabe duda de que los científicos y los hombres de negocios son quienes más ventajas sacan del manejo de dos o más idiomas, pues a pesar de pertenecer a países y culturas distintas pueden entenderse con facilidad y potenciar sus conocimientos. Por eso, es imprescindible que los profesionales y científicos de los países subdesarrollados aprendan también a comunicarse en los idiomas de los países tecnológicamente más avanzados y, sobre todo, en inglés. Así, podrán obtener provecho de los nuevos conocimientos que se difunden en la comunidad internacional, a la par de sus colegas de los países más desarrollados.

Una manera de propiciar el aprendizaje de un segundo idioma, a la que se recurre con frecuencia en el ámbito internacional, es obligando a los estudiantes universitarios a adquirir dominio de un idioma extranjero -por ejemplo, inglés o francés- como requisito para la graduación o titulación.

i) Masificar el uso de las tecnologías de información computarizadas, como la Internet, en todos los rincones de los países subdesarrollados
Nunca en la historia científica de la humanidad se ha estado tan cerca de una información inmediata sobre los acontecimientos trascendentes o los últimos avances como está ocurriendo hoy en día gracias a la magia de la Internet y la televisión por cable. Se puede decir que ya casi no hay limitación alguna en la comunicación, pues cualquier persona puede hacer conocer su última ocurrencia a todo el mundo a través de una computadora que hará llegar su información al instante a cualquier rincón del planeta.

Y ésa es una ventaja que puede ayudar a dar saltos cualitativos en el conocimiento a jóvenes escolares, universitarios, profesionales o científicos, sin necesidad de trasladarse a las «mecas» o centros famosos por su desarrollo en el campo de la investigación y el conocimiento. Gracias a la tecnología computarizada, hasta en los lugares más remotos se puede asistir y participar en conferencias de alto nivel científico.

Para el aprovechamiento adecuado de toda estas posibilidades maravillosas, sin embargo, se requiere un esfuerzo del Estado y de entidades privadas con el propósito de dotar de cabinas públicas de Internet a las provincias más lejanas y desprovistas, ubicadas generalmente en el interior de los países. Además, hay que insistir en lo señalado líneas arriba acerca de que gran parte de la información científica se difunde en inglés. De ahí la necesidad de que los educandos, profesionales y científicos lleguen a dominar este idioma.

j) Desarrollar una política de apoyo a la publicación de investigaciones o tratados de interés científico 
A pesar de sus esfuerzos de creación, uno de los dramas más frecuentes de la comunidad intelectual en los países subdesarrollados es la carencia de medios suficientes donde publicar sus artículos o escritos, por muy modestos que sean.

Este hecho es un cuello de botella que dificulta la adecuada comunicación entre los científicos, pues por falta de recursos para editar revistas o boletines, terminan guardando en sus escritorios lo que les puede haber llevado mucho esfuerzo de pensamiento e investigación. Además, impide la discusión con colegas o la confrontación de ideas, razón por la cual los científicos no evolucionan con suficiente rapidez en sus hipótesis. Así, la ciencia y tecnología quedan muertas; la imposibilidad de la publicación desestimula el trabajo y la creación.

Dado que las publicaciones científicas no son comerciales y están dirigidas a un mundo muy selectivo, la única posibilidad de que se editen e impriman pasa por conseguir apoyo financiero del Estado o entidades privadas. Es decir, a pesar de que pueden ser vendidas y, en algunos casos, contener avisos publicitarios en sus páginas, requieren generalmente de un subsidio para alcanzar cierta regularidad; de lo contrario terminan siendo «flor» de unos pocos números hasta quedar en el olvido. Y con ello los investigadores pierden un gran vehículo de información y conocimiento que les permite actualizarse cada cierto tiempo.

La posibilidad de ser leído mediante la publicación de sus trabajos de investigación en revistas o boletines es la fase final de un proceso de investigación que el científico inicia, las más de las veces, mucho tiempo atrás. De ahí que cuando no ocurre, se puede llegar a sentir la frustración del tiempo perdido, pues se pierde la oportunidad de la retroalimentación y el juicio crítico de la comunidad de científicos. La falta de ese intercambio de ideas desmotiva la productividad intelectual.

Hay que tener en cuenta que una política de promoción de publicaciones de artículos científicos, a través de revistas o libros, pasa necesariamente por un comité científico evaluador de la calidad del material, el cual debe cumplir con un mínimo de requisitos en cuanto a procedimientos de rigurosidad metodológica. En este sentido, publicar en una revista científica caracterizada por la rigurosidad o calidad de los artículos seleccionados es parte del proceso de estímulo y formación de los investigadores.

Hoy en día, si bien la Internet facilita el acceso a los materiales escritos, no logra reemplazar el valor de una impresión bien estructurada salida de la imprenta, que posee un valor intrínseco y garantiza la permanencia histórica del texto en las bibliotecas. Las publicaciones en la Internet tienden a ser temporales, sin contar con que «bajar» los textos e imprimirlos -sobre todo cuando son documentos extensos y contienen gráficos en colores-, además de caro, suele ser una tarea engorrosa cuyo resultado dista de la nitidez alcanzada por trabajo original de imprenta.

\section{Conclusiones}

- A lo largo del texto hemos tratado de argumentar en el sentido de que no basta con tener fondos importantes para dedicar a la investigación y creer 
que con ello se resuelve prácticamente el desarrollo de la ciencia y tecnología. La hipótesis o idea de trabajo del razonamiento expuesto aquí precisa que si bien el apoyo financiero es fundamental, no lo es todo, pues existen otros factores estructurales presentes en la dinámica de un país, y dentro de esa dinámica se originan las posibilidades de un desarrollo científico.

- Es innegable que sin apoyo económico no es posible la investigación ni el equipamiento de laboratorios o materiales necesarios para llevarla a cabo. Pero más allá de esos elementos indispensables para el trabajo científico, la práctica de la investigación requiere de la forja de una comunidad científica institucionalizada, con sus propias normas de trabajo y valores éticos, y cuya labor merezca el respeto de la sociedad civil y la clase política.

- Parte importante de ese respeto es el estatus o reconocimiento que la sociedad le otorga al científico y que, sin lugar a dudas, influye tremendamente como factor motivacional. Mientras mayor sea el reconocimiento a la labor científica -incluyendo salarios dignos-, más estable será el científico en su actividad y más firme su proyección hacia el futuro.

- La investigación y el desarrollo tecnológico deben guardar relación con las demandas y necesidades de las diferentes ramas de la economía y los servicios sociales de un país. Esto significa que no se puede seguir un camino trazado solamente por la curiosidad personal del científico, sino que la labor de éste debe sintonizar con el interés y las prioridades nacionales.
- El desarrollo de la industria y demás actividades propias de una economía capitalista debe marchar paralelo con el impulso a las investigaciones científicas y tecnológicas. Existe una fuerte relación entre las actividades económicas y la ciencia y tecnología, pues a mayor desarrollo de las primeras, mayor es también la demanda dirigida a que los científicos e investigadores resuelvan problemas de la producción o mejoren los niveles alcanzados. En consecuencia, es difícil el desarrollo científico o tecnológico en los países que no logran liberar las fuerzas necesarias para el desarrollo industrial y la economía de mercado.

- El potencial científico y creador de un país empieza desde la escuela. Allí se empieza a razonar con creatividad y audacia. Pero esta práctica de aprendizaje no debe ser sólo de una élite educativa, sino que debe abarcar toda la masa escolar, de manera que ella sea la base de un número suficiente de futuros científicos que desarrollan su vocación desde niños.

Sin embargo, con el propósito de obtener una masa crítica de científicos que produzcan conocimientos en cantidad suficiente como para independizar un país en el campo de la investigación científica y tecnológica, se requiere consolidar también la educación postescolar o universitaria, especialmente la de postgrado. Un país que no promueve a los profesionales con vocación investigadora de modo que sigan estudiando y obteniendo los más altos grados académicos (doctor), ni los auspicia con becas o investigaciones postdoctorales, no puede llegar a obtener científicos de calidad. A lo más logrará una masa pensante que no estará a la altu- 
ra de los investigadores de los países más avanzados. De ser así, la distancia entre países subdesarrollados y desarrollados no se acortará.

\section{Recomendaciones}

Actuar desde hoy pensando en el futuro es tener capacidad prospectiva. Y en lo referente al tema de la ciencia y tecnología, es claro que estamos ante el factor que revoluciona día a día la capacidad productiva, la generación de riqueza y las posibilidades de bienestar de la población. Más aún, es ahora el factor de mayor valor agregado y posiblemente sea el más importante para el futuro de la humanidad; hecho que se expresa sobre todo en el campo de la creación de inteligencia artificial. En la actualidad, las empresas que obtienen mayores ganancias son las que producen equipos y lenguajes sistémicos de computación. Si se quiere lograr eficiencia, no hay campo en la actividad económica, servicios públicos e incluso economía doméstica que pueda prescindir de estas herramientas y conocimientos.

¿Qué deben hacer los países atrasados para no quedarse a la zaga de los países industrializados?

Como se ha visto, el motor que debe empujar el desarrollo de la ciencia y tecnología tiene varios componentes. Ellos deben funcionar paralelamente y con armonía para evitar desbalances que hagan flaquear al sistema.

La única manera de que todo funcione bien desde el comienzo es pensar en el país como algo integral, dentro del cual haya un rol asignado a la ciencia y tecnología. Para ello es imprescindible elabo- rar un plan nacional de desarrollo que, entre las reformas o actividades importantes por acometer, comprenda las relacionadas con el impulso a la investigación científica y la creación tecnológica. Pensar por separado en este factor, sin tomar en cuenta la marcha del resto de la sociedad, como el sistema educativo y las posibilidades del desarrollo industrial, entre otros aspectos ya mencionados, equivale a concebir el problema con una visión parcial que, obviamente, llevará también a recetas parciales.

Es probable que, a muchas personas, la idea de un plan nacional de desarrollo les suene a dirigismo estatal, pero éste es también un prejuicio que debe evitarse. El concepto de desarrollo, sea científico-tecnológico o económico-social, hace referencia a la actuación armoniosa de un conjunto de elementos que se condicionan o refuerzan entre sí con el propósito de lograr ciertos objetivos y metas. Y esto requiere un manejo fino y coordinado de políticas para los diversos sectores del país. Si se dejara el comportamiento de los diversos sectores productivos y no productivos a la espontaneidad o al azar, se obtendría permanentemente desequilibrios y pérdida de recursos y tiempo.

Un plan nacional de desarrollo dentro del cual se incluyan políticas específicas orientadas al impulso de la ciencia y tecnología, y que prevea el impacto y la interacción de éstas con los otros campos de la realidad nacional, es un instrumento que los países atrasados deben usar para lograr un desarrollo pronto y armonioso. Dentro de este contexto será posible orientar el apoyo a las actividades de investigación en función de la demanda, actual y futura, del resto de sectores productivos y no productivos de la vida nacional. 